\title{
Origin of ion cyclotron emission at the proton cyclotron frequency from the core of deuterium plasmas in the ASDEX-Upgrade tokamak
}

\author{
B Chapman ${ }^{1,2}$, R O Dendy ${ }^{1,2}$, S C Chapman ${ }^{2}$, K G \\ McClements $^{1}$, R Ochoukov ${ }^{3}$ \\ ${ }^{1}$ CCFE, Culham Science Centre, Abingdon, OX14 3DB, United Kingdom of Great \\ Britain and Northern Ireland \\ ${ }^{2}$ Centre for Fusion, Space and Astrophysics, University of Warwick, Coventry, CV4 \\ 7AL, United Kingdom of Great Britain and Northern Ireland \\ 3 Max Planck Institute for Plasma Physics, Boltzmannstr. 2, D-85748, Garching, \\ Germany \\ E-mail: benjamin.chapman@ukaea.uk
}

April 2020

\begin{abstract}
Observations have recently been made of ion cyclotron emission (ICE) that originates from the core plasma in the DIII-D and ASDEX-Upgrade tokamaks. In some of these cases, the ICE spectral peaks correspond to the local cyclotron harmonic frequencies of fusion-born ions close to the magnetic axis. This is in contrast to the hitherto usual spatial localisation of the ICE source to the outer midplane edge in tokamak and stellarator plasmas. Here we show that a possible emission mechanism for core ICE in ASDEX-Upgrade deuterium plasmas can arise from the rapid onset and rise of local fusion reactivity. This would give rise to a transiently highly non-Maxwellian population of fusion-born protons near their birth energy, prior to collisional slowingdown on longer timescales. Such populations are often liable to fast radiative relaxation under the magnetoacoustic cyclotron instability which underpins ICE, depending also on bulk plasma parameter values. We therefore perform first principles computations of the self-consistent collective relaxation of such a population, using a particle-in-cell code, for plasma parameters appropriate to the ASDEX-Upgrade core. The resulting simulated ICE spectra include a strong peak at the proton cyclotron frequency that corresponds well to the observations.
\end{abstract}

PACS numbers: 52.35.Hr, 52.35.Qz, 52.55.Fa, 52.55.Tn

Keywords: ion cyclotron emission, magnetoacoustic cyclotron instability, tokamak, numerical simulation, particle-in-cell, bispectral analysis 


\section{Introduction}

Strongly suprathermal radiation known as ion cyclotron emisison (ICE) is widely observed in magnetic confinement fusion (MCF) plasmas $[1,2,3,4,5,6,7,8,9$, $10,11,12,13,14,15,16,17,18,19,20,21,22,23]$. Its intensity is typically orders of magnitude greater than that of black-body radiation from thermal ions, and its spectral peak frequencies correspond to multiple cyclotron harmonics of one or more energetic ion species at a specific radial location. The excitation mechanism for ICE is the magnetoacoustic cyclotron instability (MCI) [24, 25, 26, 27, 28, 29, 30, 31, 32, 33, 34], which can occur at locations where a minority energetic ion population has a velocityspace distribution which has the property of an inversion i.e. where $\partial f / \partial v>0$. Under these circumstances, wave-particle cyclotron resonance occurs with waves on the fast Alfvén-cyclotron harmonic branch which are supported by the bulk plasma and propagate nearly perpendicular to the background magnetic field. It is also possible for ICE to be driven by anisotropy in the minority energetic ion distribution [30, 35]. These energetic ions can originate from fusion reactions in the core plasma, neutral beam injection (NBI), or heating due to externally injected waves in the ion cyclotron range of frequencies (ICRF). In general, there will be a link between the spectrum of waves excited by the MCI and the character of the velocity distribution of the energetic ions which drive the waves. Mapping between the measured ICE signal and the theoretical characteristics of the MCI-excited waves is thus essential to interpreting the energetic ion physics underlying ICE observations.

In recent years, the MCI has been extensively studied computationally using particle-in-cell (PIC) computations [36, 37, 38, 39, 40, 41, 42, 43, 44, 45]. These PIC computations use kinetic plasma species to resolve the full gyro-orbit ion dynamics, evolving self-consistently with the electric and magnetic fields under the MaxwellLorentz equations. These can carry the MCI instability into its nonlinear regime, and this is essential when interpreting some key aspects of ICE observations [36, 41, 43].

Most of the early ICE observations were localised to the outer midplane edge region of the MCF plasmas $[1,2,3,4,5,6,7,11,19,20]$, but recently ICE has also been detected from the core plasmas of ASDEX-Upgrade [8, 9, 10] and DIII-D [12, 13, 14]. Figure 5a of Ref. [8] (repeated here as Fig. 1) shows an example of central ICE at the fundamental proton cyclotron harmonic (equal to the second deuteron cyclotron harmonic) with $f \approx 39.6 \mathrm{MHz}$ in ASDEX-Upgrade. It can be seen that the start of the transient ICE signals is correlated with the NBI switch on time. This deuterium plasma was heated by deuterium NBI with injection energy $60 \mathrm{keV}$, and therefore contained both energetic fusion-born protons created at $3.0 \mathrm{MeV}$ and energetic NBI deuterons. The Alfvén velocity in the core ASDEX-Upgrade plasma was 5 to $6 \times 10^{6} \mathrm{~ms}^{-1}$; this value is lower than the proton birth velocity $\left(2.4 \times 10^{7} \mathrm{~ms}^{-1}\right)$, but is a factor of two above the NBI deuteron velocity $\left(2.4 \times 10^{6} \mathrm{~ms}^{-1}\right)$ at injection. Previous studies have shown $[36,37,38,44,45]$ that the MCI is more readily excited for $v_{\perp} / v_{A} \gtrsim 1$, where $v_{A}$ is the local Alfvén speed. For this reason, it was concluded [8] that the most likely 
driver of this ICE is fusion-born protons.

Similar core ICE, also correlated with the time evolution of the NBI power, was recently observed [10] in pure hydrogen plasmas in which no fusion-born protons are present. Combining linear analytical calculations [27, 30] with these further experimental ICE results led to the conclusion that this form of core ICE at the fundamental proton cyclotron harmonic (equal to the second deuteron cyclotron harmonic) in ASDEX-Upgrade can be explained in terms of a driving population of sub-Alfvénic NBI deuterons. This conclusion does not preclude fusion-born protons from being the driver of core ICE in deuterium discharges. Indeed the present study is motivated by our conjecture that increased fusion reactivity caused by NBI switch on could explain the correlation in deuterium plasmas. It is noteworthy that emission at the deuteron cyclotron frequency (i.e. the fundamental harmonic) was not observed from this particular deuterium plasma. This contributes to the present study, where we revisit the possibility that the MCI of fusion-born protons is the driving mechanism behind the core ICE reported in Ref. [8].

It is possible that both energetic ion populations are contributing to the observed emission at the fundamental proton (second deuteron) cyclotron harmonic. The relative linear drive of these two mechanisms depends primarily on two things: the ratio $v_{\perp} / v_{A}$ and the fast particle concentration $\xi=n_{f p} / n_{D}$, where $n_{f p}$ is the density of the energetic ions and $n_{D}$ is the density of the background ions. The number of NBI ions is expected to be much greater than the number of fusion-born protons, however, as discussed above, $v_{\perp} / v_{A}$ is much higher for the fusion-born proton population than for an NBI deuteron population. The NBI deuteron population is sub-Alfvénic, but the fusion-born protons are super-Alfvénic, meaning they are much more capable of generating ICE via the linear MCI. These considerations apply only to the linear drive of MCI excited ICE harmonics. As we shall show, it is the nonlinear stage of the MCI that is responsible for fusion-born proton driven ICE at the fundamental proton cyclotron frequency. By carrying out particle-in-cell (PIC) [46] computations using one spatial dimension and three velocity dimensions (1D3V) which extend deep into the nonlinear stage of the MCI, we show that emission at the fundamental proton cyclotron frequency can be explained, provided nonlinear interactions are taken into account. In section 2 we describe our simulation set-up, including the EPOCH [47] PIC code and the choice of distribution function to represent the initial configuration of the fusion-born protons in velocity space. In section 3 we discuss the findings of our simulation, with a particular emphasis on the nonlinear dynamics. We discuss our results in relation to recent experiments in section 4.

\section{Computational approach and simulation details}

The birth distribution of fusion-born ions in velocity space is a spherically symmetric shell. In a tokamak plasma, this will be anisotropically depleted by the prompt loss of ions born on unconfined orbits, on microsecond timescales longer than the ion gyro period. Collisional effects will populate the initially hollow sphere within the shell with 


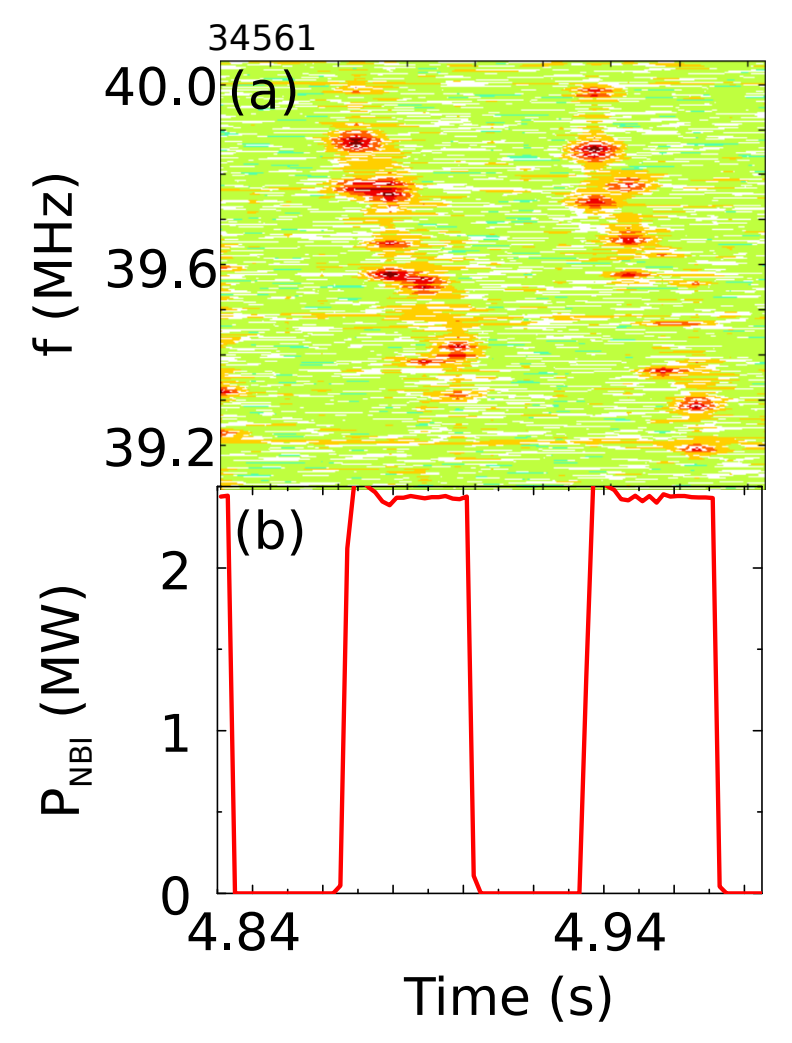

Figure 1: a) Spectrogram showing the measured ICE spectra from ASDEX-Upgrade deuterium plasma \#34561 heated by repeated transient bursts of deuterium NBI with $60 \mathrm{keV}$ injection energy. The observed emission is at the proton cyclotron frequency (equal to the second deuteron cyclotron harmonic) and exhibits fine stucture which is discussed in more detail in Ref. [8]. b) Time evolution of NBI power, with which the observed ICE is correlated. Reprinted from Review of Scientific Instruments 89, 10J101 (2018), AIP Publishing.

slowed-down fusion-born ions, so that their velocity distribution becomes monotonically decreasing on collisional timescales. For the ASDEX-Upgrade core plasma we study here, the slowing down time is $\sim 200 \mathrm{~ms}$. At early times after the onset of significant fusion reactivity, and before collisions have had time to take effect, the velocity-space distribution of fusion-born ions will exhibit a population inversion which approximates a spherical shell, subject also to incipient prompt losses. The true velocity distribution on sub-collisional timescales $(\lesssim 200 \mu s)$ after fusion onset can therefore be approximated, for present purposes, by a velocity-space structure somewhere between a ring-beam and a shell. We note that the duration of the NBI bursts, and hence the duration of ICE spectra is $\sim 40 \mathrm{~ms}$, significantly less than the fusion-born proton slowing down time. Recent work [43] has shown that ring-beam simulations can act as a reliable proxy for shell simulations, while requiring only a fraction of the computational resources. To this end, we carry out a simulation of ICE excitation via the MCI in which the initial distribution in velocity space of the fusion-born protons is represented analytically as a 
ring-beam,

$$
f\left(v_{\|}, v_{\perp}\right) \propto \exp \left(\frac{-\left(v_{\perp}-v_{0 \perp}\right)^{2}}{v_{T \perp}^{2}}\right) \delta\left(v_{\|}\right) .
$$

Here $v_{\|}$and $v_{\perp}$ are the parallel and perpendicular velocity components respectively, $v_{0 \perp}$ is the initial perpendicular velocity, and $v_{T \perp}$ is the perpendicular velocity spread, i.e. the width of the ring.

We use the EPOCH particle-in-cell (PIC) code [47] to self-consistently solve the Maxwell-Lorentz system of equations along with the relativistic Lorentz force law, for tens of millions of particles. Our computational domain spans one periodic (closed) spatial dimension and all three velocity dimensions (1D3V), and the code captures the full gyro-orbit particle dynamics of the electrons background deuterons, and the minority energetic proton population, while self-consistently evolving all three vector components of the electric and magnetic fields. The thermal deuterons and electrons in our system are initialised with Maxwellian velocity distributions. The fully kinetic physics that ensues as these three particle species interact with the self-consistently generated electric and magnetic fields corresponds to the MCI. We allow our simulation to progress deep into the nonlinear regime and reach saturation. By using Fourier transforms in the post-processing stage, we are to identify both linearly and nonlinearly excited waves, which are the result of the MCI physics unfolding within the MaxwellLorentz system. The simulation is set up in slab geometry, corresponding to the local approximation; it does not incorporate any toroidal effects or eigenmode structure $[32,33,34,48,49,50,51,52,53,54,55]$. The omission of toroidal effects is expected to have only marginal consequences for modelling the ICE phenomenology addressed here, given the success of calculations and computations that use the local approximation in explaining results from JET and TFTR $[1,2,4,3,7,38,36]$, as well as more recent ICE observations from KSTAR [40, 41, 42] and LHD [56, 44].

The fusion-born protons are initialised with $v_{\perp 0} \simeq 7.58 \times 10^{6} \mathrm{~ms}^{-1}$ giving $v_{\perp 0} / v_{A} \sim$ 1.16. In Ref [8], the Orb and Dimon fast ion full orbit tracking codes were used to determine the region of velocity space in which fusion protons born at the magnetic axis remained on confined orbits. Denoting the total velocity by $|\mathbf{v}|$, it was determined that fast ions remain confined when they have pitch values $v_{\|} /|\mathbf{v}|>0.45$ and $v_{\|} /|\mathbf{v}|<-0.72$ for co- and counter- current orbits respectively. Our choice of $v_{\perp 0}$ is consistent with this, giving $\left|v_{\|}\right| /|\mathbf{v}| \approx 0.95$. This is also in line with the findings of Ref. [40], in which a subset of the fusion-born protons in KSTAR (a tokamak with a similar size to ASDEX-Upgrade) were found to remain on confined orbits when $\left|v_{\|}\right| /|\mathbf{v}| \approx 0.96$. Let us summarise the physical motivation for our choice of $v_{\perp}$. First it is of the order $v_{A}$, which previous work has shown maximises the drive of the MCI [36, 37, 38, 44, 56]. Second, it is consistent with the results of the Orb and Dimon full orbit tracking codes for this pulse. Third, in combination with $v_{\|}$it yields a pitch angle which allows for a confined orbit. We are able to neglect the parallel component of the fusion-born proton velocity $v_{\| 0}$ for the following reason. If an energetic ion has a velocity component parallel to the magnetic 
field, cyclotron resonance in its rest frame is Doppler-shifted (through $k_{\|}$) with respect to the cyclotron resonance of the majority ions in their rest frame. This frequency shift can switch off cyclotron damping by the majority ions, of the waves preferentially excited by this energetic ion. For $k_{\|} \sim 0$ as in our simulations, the Doppler shift will always be negligible, and the choice of $v_{\| 0}$ is therefore inconsequential. We introduce a $10 \%$ perpendicular velocity spread $v_{T \perp}$, which has the effect of decreasing the linear growth rate of linearly unstable modes, resulting in a slightly longer simulation time and improved frequency resolution [43]. Our simulation uses a background magnetic field $B_{z}=2.62 \mathrm{~T}$ in line with experiment, which is oriented at $89^{\circ}$ to the onedimensional spatial domain of the simulation. The thermal plasma comprises initially Maxwellian deuterons and electrons, with temperature $T_{D}=T_{e}=5 \mathrm{keV}$. The electron number density $n_{e}=3.8 \times 10^{19} \mathrm{~m}^{-3}$, and the ratio of energetic to background ions $\xi=n_{p} / n_{D}=10^{-3}$. Such a large concentration is not realistic in tokamak plasmas, but is necessary to obtain adequate signal-to-noise ratios in a feasible amount of computational time. Previous computational studies [37] have shown that the simulated ICE power scales linearly with fast particle concentration $\xi$, implying that our results would be qualitatively unchanged at lower concentrations.

To establish contact with experiment, our PIC simulation must excite ICE at the fundamental proton cyclotron harmonic, which is a challenging task. Previous simulations and analysis of the MCI in related contexts have shown that the fundamental, along with other harmonics with $\omega \lesssim 6 \omega_{c p}$ where $\omega_{c p}$ is the proton cyclotron frequency, are typically linearly stable. They are often driven exclusively by nonlinear interactions among linearly unstable MCI excited modes that have $\omega \gtrsim 6 \omega_{c p}$. Examples are shown in Fig. 3 of Ref. [43] and Fig. 3a of Ref. [36]. This nonlinear drive is important for matching the full spectral extent of ICE between simulations and experiment, and is naturally weaker than the linear MCI drive at higher harmonics. This, combined with the particle noise present in the low frequency region of PIC simulations, means that the PIC simulation we report here is necessarily highly resolved and therefore computationally intensive. The simulation uses a total of 38,000 grid cells which fully resolve the Debye length, with 4,000 particles per cell and higher order particle weighting functions. To quantify the effect of particle noise due to thermal plasma fluctuations, a companion simulation is performed without the minority fusionborn protons.

\section{Analysis of simulation outputs}

We first consider the flow of energy in our MCI simulation, which is plotted in Fig. 2. This resembles that in previous work [38, 36, 42, 43]: the minority protons transfer their energy predominantly to the z-component of the fluctuating magnetic field, as well as to the thermal deuterons. This reflects the fast Alfvénic character of the excited waves. The simulation is run for eighteen proton gyro-periods $\tau_{g p}$ and the linear phase of the MCI reaches saturation by $t \approx 10 \tau_{g p}$. Thereafter the MCI enters its nonlinear phase [36]. 
We will show that the plasma dynamics in this stage give rise to nonlinear excitation of a peak at the fundamental proton cyclotron harmonic in the simulated ICE spectrum.

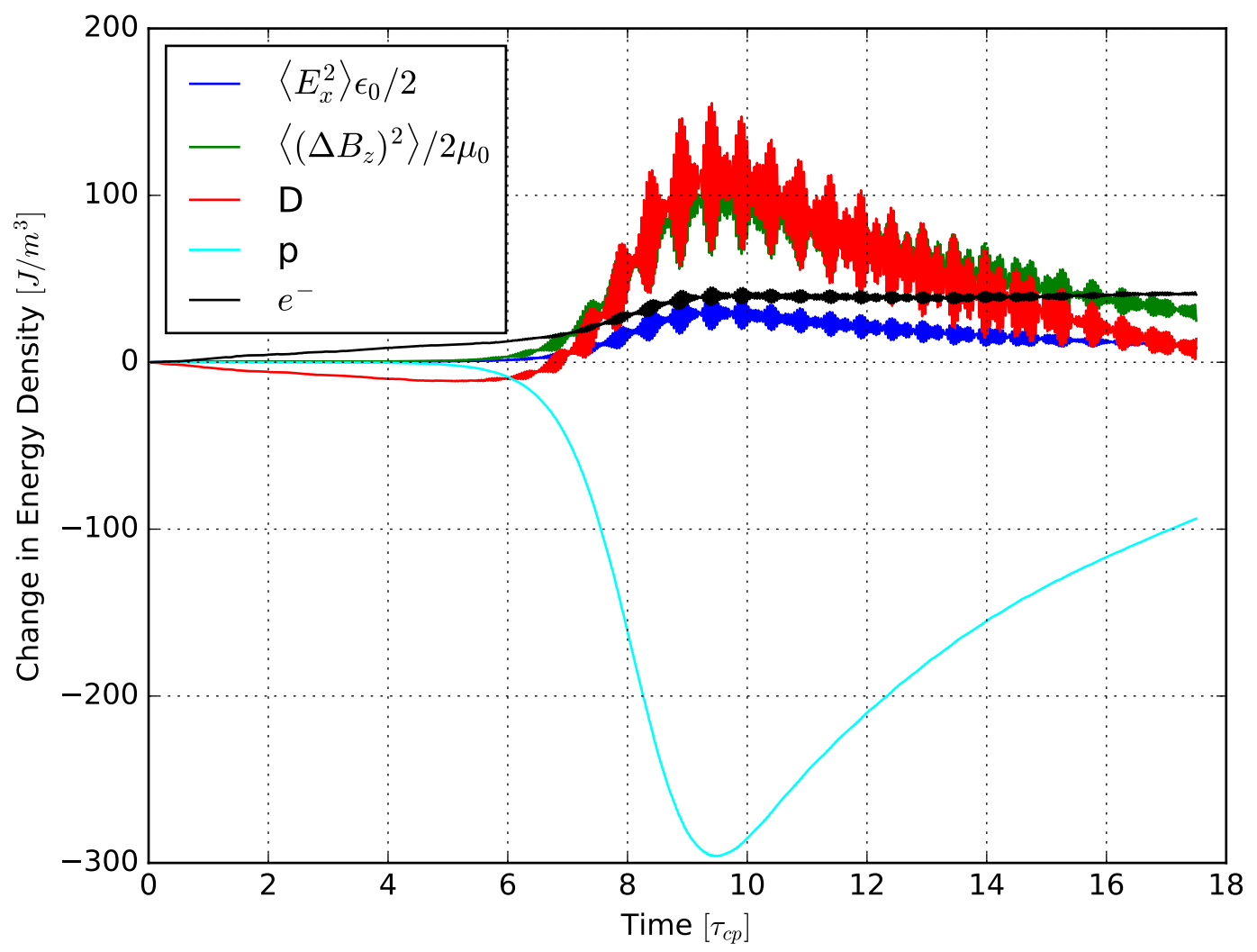

Figure 2: Time evolution of the change in energy density of particles and of electric and magnetic field components. The traces, ordered from top to bottom at their peak (and in colour online) are: Top (red) the change in kinetic energy density of the thermal bulk plasma deuterons; second (green) the energy density of the magnetic field perturbation $\Delta B_{z}$; third (black) the change in kinetic energy density of the thermal bulk plasma electrons; fourth (blue) the energy density of the electrostatic field $E_{x}$; fifth (magenta) the energy density of the $y$-component of the magnetic field $B_{y}$; sixth (cyan) the change in kinetic energy density of the minority energetic protons. Time is normalised to the proton gyro-period $\tau_{c p}$. The linear phase of the MCI saturates at $t \sim 9.5 \tau_{c p}$.

Figure 3 shows the power spectrum of the fluctuating $\Delta B_{z}$ field component as a function of frequency $\omega$, plotted on a $\log _{10}$ scale. This plot is a temporal Fourier transform over the first $10 \tau_{g p}$ of the simulation, up to the linear phase, after integration across the entire spatial domain. Here, the blue trace denotes the power in the MCI simulation, while the green trace denotes the baseline power in the background thermal plasma with no energetic protons present. Spectral peaks at proton cyclotron harmonics in the range $7 \omega_{c p} \leq \omega \leq 15 \omega_{c p}$ are promptly excited (see also Fig. 2); the strongest peaks at $\omega=11 \omega_{c p}$ and $\omega=12 \omega_{c p}$ are more than five orders of magnitude more powerful than the background plasma signal. Modes with $\omega \leq 6 \omega_{c p}$ appear to be 
linearly stable, and their power in the MCI simulation is approximately equal to the power in the thermal plasma. Due to the high number of computational macroparticles in our simulation, we observe residual spectral structure at these low harmonics, which reflects the concentration of noise energy at normal modes in line with the fluctuationdissipation theorem [46]. The time evolution of $\Delta B_{z}$ as a function of wavenumber is plotted in Fig. 4. For quasi-perpendicular wave propagation in the ion cyclotron range of frequencies, we benefit from an approximately one-to-one mapping between wavevector and frequency, so that Fig. 4 yields information about the spectral energy content at proton cyclotron harmonics as a function of time. The dominant modes with $k \geq 8 \omega_{c p} / V_{A}$ are clearly linearly unstable, and correspond to the peaks with $\omega \geq 8 \omega_{c p}$ in Fig. 3. There are three further striking features of this plot., which arise for $t \gtrsim 8 \tau_{c p}$ : the modes at $k \simeq 1.2 \omega_{c p} / V_{A}, k=2.5 \omega_{c p} / V_{A}$, and $k=7.1 \omega_{c p} / V_{A}$, which correspond to $\omega \simeq 1 \omega_{c p}, \omega \simeq 2 \omega_{c p}$, and $\omega \simeq 7 \omega_{c p}$ respectively. The direct mapping between $k$ and $\omega$ is a result of the structure of the dispersion relation shown in the left panel of Fig. 5 in which $|\mathbf{k}| \approx k_{\perp}$ and $k_{\|} \approx 0$. We shall show below that these modes are driven almost entirely by nonlinear wave-wave interactions involving the modes with larger amplitude that are linearly unstable from the outset, with spectral peak frequencies in the range $8 \omega_{c p} \lesssim \omega \lesssim 15 \omega_{c p}$.

Figure 5 plots the spatio-temporal Fourier transform calculated using data from the full $17.5 \tau_{g p}$ duration of the simulation. The left panel shows a wide range of frequencies and wavenumbers over which the nonlinear interactions manifest as spectrally dense regions close to, but not quite the fast Alfvén branch, which are spread across a wide range of Fourier space. The right panel of Fig. 5 is a close up of the low frequency modes in the simulation. We see that the two nonlinearly driven modes at normalised $k=1.2$ and $k=2.5$ lie just to the right (left) of the forward (backward) propagating fast Alfvén wave.

Using Fourier transform windows that have different temporal durations, we can further quantify the difference between the MCI and background simulations. Figure 6 plots the difference in the $\log _{10}$ of power in the $\Delta B_{z}$ component as a function of frequency. We focus on the low frequency region $\omega \leq 6 \omega_{c p}$, corresponding to the linearly stable modes shown in Fig. 3. Panels a), b), c), and d) of Fig. 6 correspond to temporal Fourier transform windows starting at $t=0$ and of duration $10 \tau_{g p}, 12.5 \tau_{g p}, 15 \tau_{g p}$, and $17.5 \tau_{g p}$ respectively.

It is clear that the spectral peaks are at proton cyclotron harmonics $\omega=\omega_{c p}$ and $\omega=2 \omega_{c p}$ increase in power as the simulation progresses deeper into the nonlinear stage of the MCI beyond $t \approx 10 \tau_{c p}$. The experimentally measured $\omega=\omega_{c p}$ peak shows the most pronounced effect: its power is about an order of magnitude higher than the background signal at the end of the simulation. We note that the power in the background plasma increases slightly as the simulation progresses. The rate of this increase is approximately constant, and is consistent across the whole range of frequencies (including proton cyclotron harmonic peaks at $\omega>6 \omega_{c p}$ not shown here) for the entire duration of the simulation. In the linear stage of the simulation, any increase 


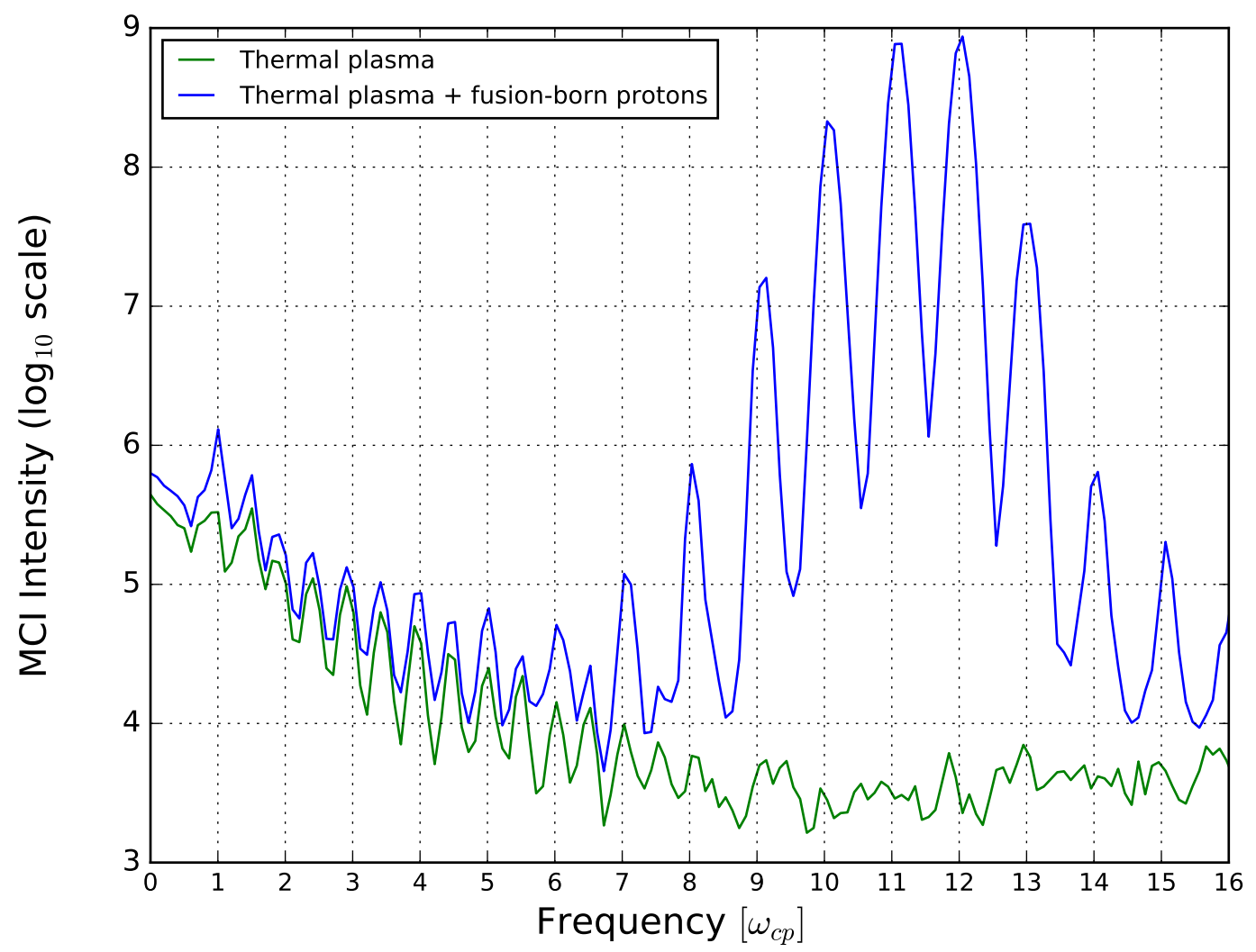

Figure 3: Blue trace: Spectral intensity of the fluctuating $\Delta B_{z}$ field energy density calculated using a time window from zero to ten proton gyro-periods, corresponding approximately to the linear phase of the MCI instability. Vertical axes are plotted on a $\log _{10}$ scale, and the horizontal axis is normalised to the minority proton cyclotron frequency $\omega_{c p}$. Green trace: Power in a background thermal plasma without a minority energetic proton ring-beam, so that any spectral structure arises from the fluctuation-dissipation theorem and identifies normal modes (i.e. no nonlinear coupling), thereby providing a noise baseline for the blue traces.

in Fourier power in modes with $\omega \leq 6 \omega_{c p}$ exactly follows that of the background thermal plasma simulation, and is therefore due only to thermal noise. To further indicate that the observed spectral power at the fundamental proton cyclotron harmonic is generated by nonlinear interactions between MCI excited modes of higher frequency, we plot the evolution in the difference between the $\log _{10}$ of the MCI and thermal plasma Fourier power at $\omega=\omega_{c p}$ as a function of time. This is shown in the left panel of Fig. 7, while the right panel replots the data shown in panels a) and d) of Fig. 6.

We observe a negligible difference in power up to $t \approx 5 \tau_{g p}$, which corresponds to the initial stage of the simulation in which the linearly unstable modes are not yet fully excited, see Fig. 4. From $t \approx 5 \tau_{g p}$ to $t \approx 12 \tau_{g p}$, we observe an approximately linear increase in the power difference, which corresponds to the linear excitation stage of the simulation (for spectral peaks with $\omega \gtrsim 8 \omega_{c p}$ ) and the beginning of the nonlinear stage of the simulation; recall also Fig. 2 . From $t \approx 12 \tau_{g p}$ onwards, deep into the nonlinear 


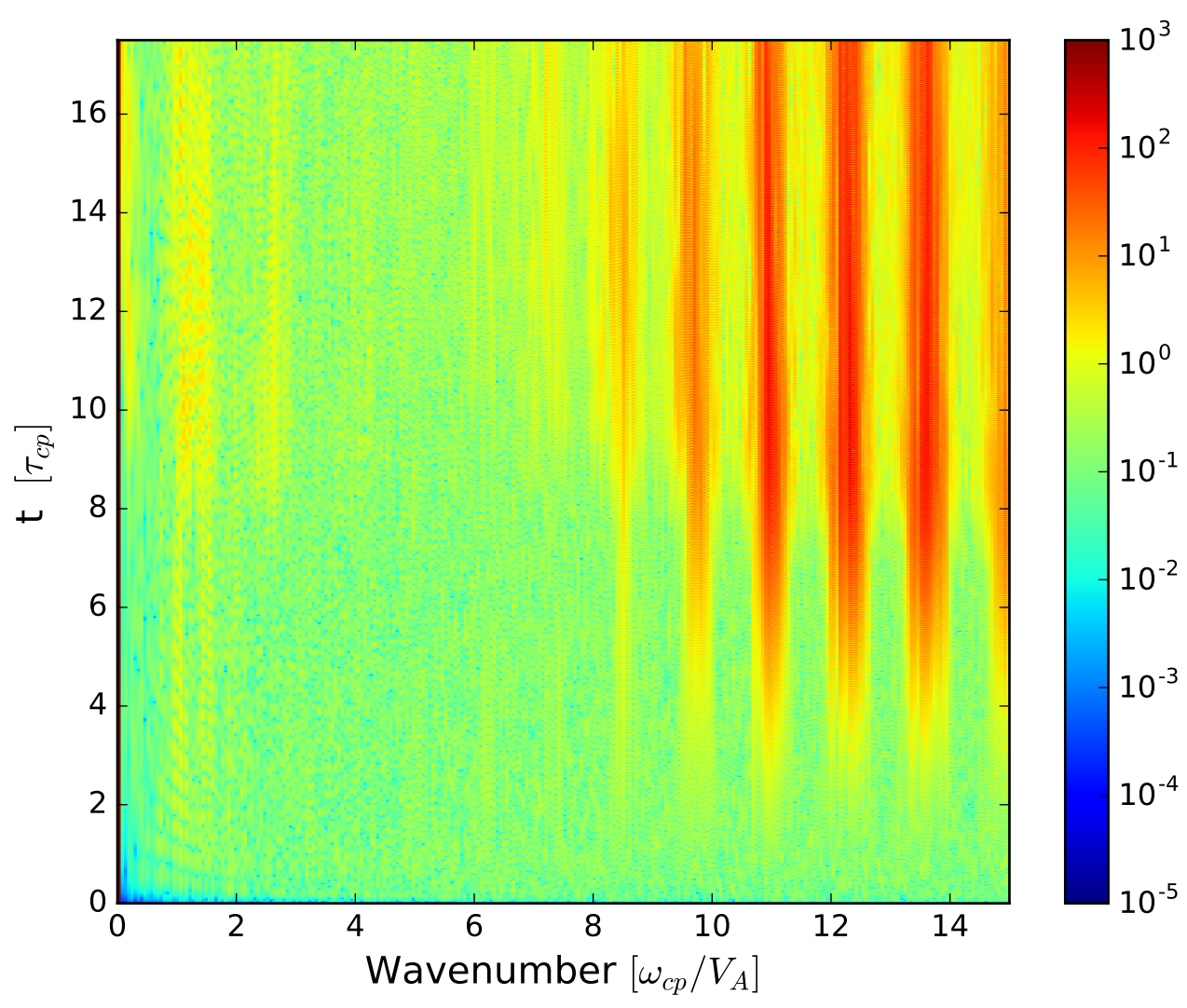

Figure 4: Time evolution of the spectral density of the fluctuating z-component of the magnetic field $\Delta B_{z}$ across wavenumber space, plotted with a $\log _{10}$ scale colour bar. The vertical axis is normalised to the minority proton gyroperiod $\tau_{c p}=2 \pi / \omega_{c p}$, and the horizontal axis is normalised to $\omega_{c p}$ divided by the Alfvén speed $V_{A}$. At least three nonlinearly driven modes at normalised $k \simeq 1.2,2.5$, and 7.1, which correspond to normalised $\omega \simeq 1,2$, and 7 , begin growing at approximately the start of the nonlinear stage of the simulation at $t \approx 9 \tau_{g p}$.

stage of the simulation, we see a rapid increase in the difference in power, reaching almost a factor of ten.

Returning for a moment to Fig. 6, we see that there is no noticeable peak present at $\omega=2 \omega_{c p}$ at the beginning of the nonlinear stage in panel a) (see also the $k \simeq 2.5$ region in Fig.4). As the simulation progresses deeper into the nonlinear stage, a peak at $\omega=2 \omega_{c p}$ manifests, and is approximately $10^{0.6} \approx 4$ times larger than the background signal by the end of the simulation at $t \approx 17.5 \tau_{c p}$. For ASDEX-Upgrade plasma \#34561 considered here (see Fig. 1), the frequency of the second proton cyclotron harmonic is above the Nyquist frequency of the detection system [8], and therefore cannot be detected reliably. However, Fig. 4 of Ref. [8] shows measured ICE spectra for a similar ASDEX-Upgrade plasma with lower magnetic field (hence lower $\omega_{c p}$ ), and a clear ICE peak at $\omega=2 \omega_{c p}\left(4 \omega_{c D}\right)$ was measured below the Nyquist frequency. In much the same way as the simulation data displayed in Fig.6, this experimentally measured peak is less 

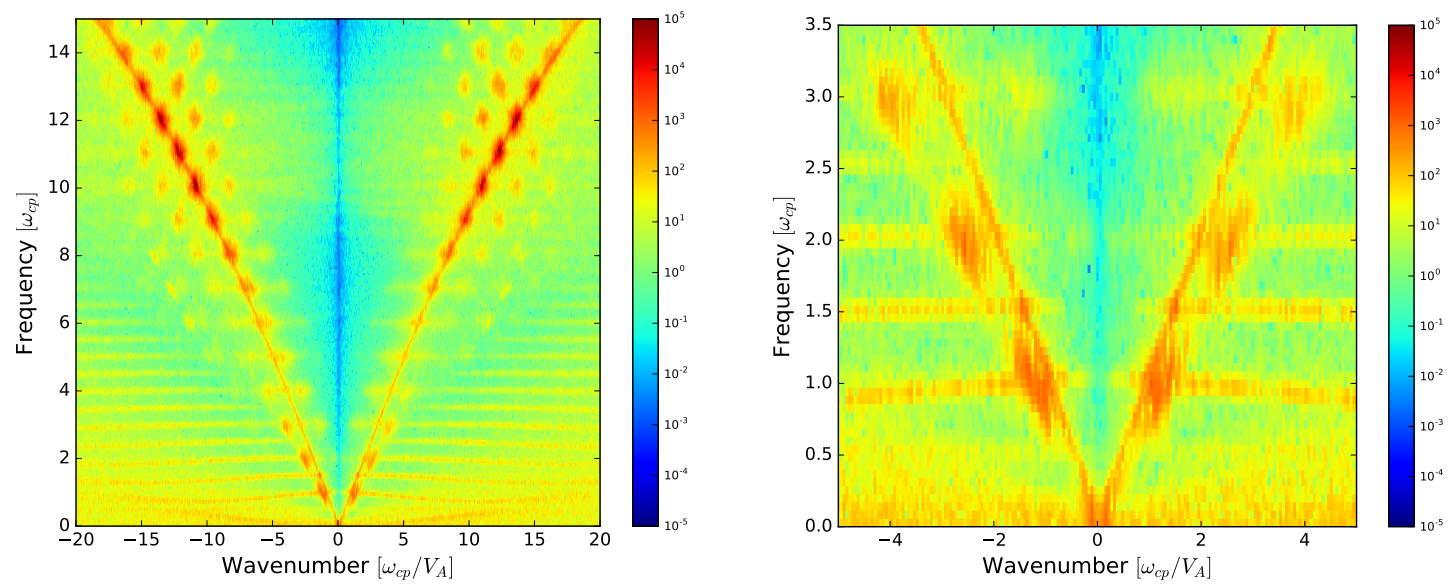

Figure 5: Spatiotemporal Fourier transform of the spectral density of the fluctuating zcomponent of the magnetic field $\Delta B_{z}$ across frequency-wavenumber space. The transform is taken across the entire spatial domain and duration shown in Fig. 2, and the colour bar indicates the $\log _{10}$ of the spectral density. The vertical axis is normalised to the minority proton cyclotron frequency $\omega_{c p}$, and the horizontal axis is normalised to $\omega_{c p}$ divided by the Alfvén speed $V_{A}$. Left: forward and backward propagating waves across the range $0 \leq \omega \leq 15 \omega_{c p}$ and $-20 \leq k v_{A} / \omega_{c p} \leq 20$. This plot is dominated by the strongly excited linearly unstable modes in the range $9 \omega_{c p} \leq \omega \leq 12 \omega_{c p}$ which lie along the fast Alfvén dispersion branch (see Fig. 3). In this frequency range there are also visible nonlinearly driven modes that are immediately adjacent to the fast Alfvén branch. Right: an expanded view of the low frequency region of the left panel. There are regions of high spectral density at proton cyclotron harmonics lying just to the right (left) of the forward (backward) propagating fast Alfvén wave. The thermal cyclotron harmonic waves at, for example, $3 \omega_{c p} / 2$ and $5 \omega_{c p} / 2$ correspond to odd harmonics of the background deuteron cyclotron frequency $\omega_{c D}=\omega_{c p} / 2$, and are normal modes of this two ion species plasma.

spectrally intense than the emission at $\omega=\omega_{c p}\left(2 \omega_{c D}\right)$.

As a final diagnostic of the nonlinear interactions in the system we plot the squared bicoherence $[57,58,59,60,61] b_{c}^{2}$ which is defined by Eq. B.2 of Ref. [41]. The value of $b_{c}^{2}$ is bounded between 0 and 1 , and measures the fraction of the Fourier power of a signal that is due to quadratic nonlinear interactions between three waves that satisfy the frequency and wavenumber matching criteria: $f_{3}=f_{1} \pm f_{2}$ and $\boldsymbol{k}_{\mathbf{3}}=\boldsymbol{k}_{\mathbf{1}} \pm \boldsymbol{k}_{\mathbf{2}}$. Bicoherence analysis is routinely applied to experimental plasma physics measurements, see for example, Refs. [62, 63, 64, 65], and has been successfully applied to ICE data and MCI simulations [36, 41, 43], and to other experimental plasma measurements. For further details see Appendix B of [41]. The squared bicoherence $b_{c}^{2}$ of the $B_{z}$ field component is plotted in Fig. 8. We compute $b_{c}^{2}$ by successively Fourier transforming in the time domain, and then plot our results in wavenumber space where we benefit from increased resolution. For the strongest modes in the simulation, which are subject to the empirical dispersion relation that is plotted in the left panel of Fig. 5, this wavenumber information maps directly to frequency. Shading in Fig. 8 indicates the intrinsic strength of nonlinear coupling, 1 (dark red) being completely coupled and 


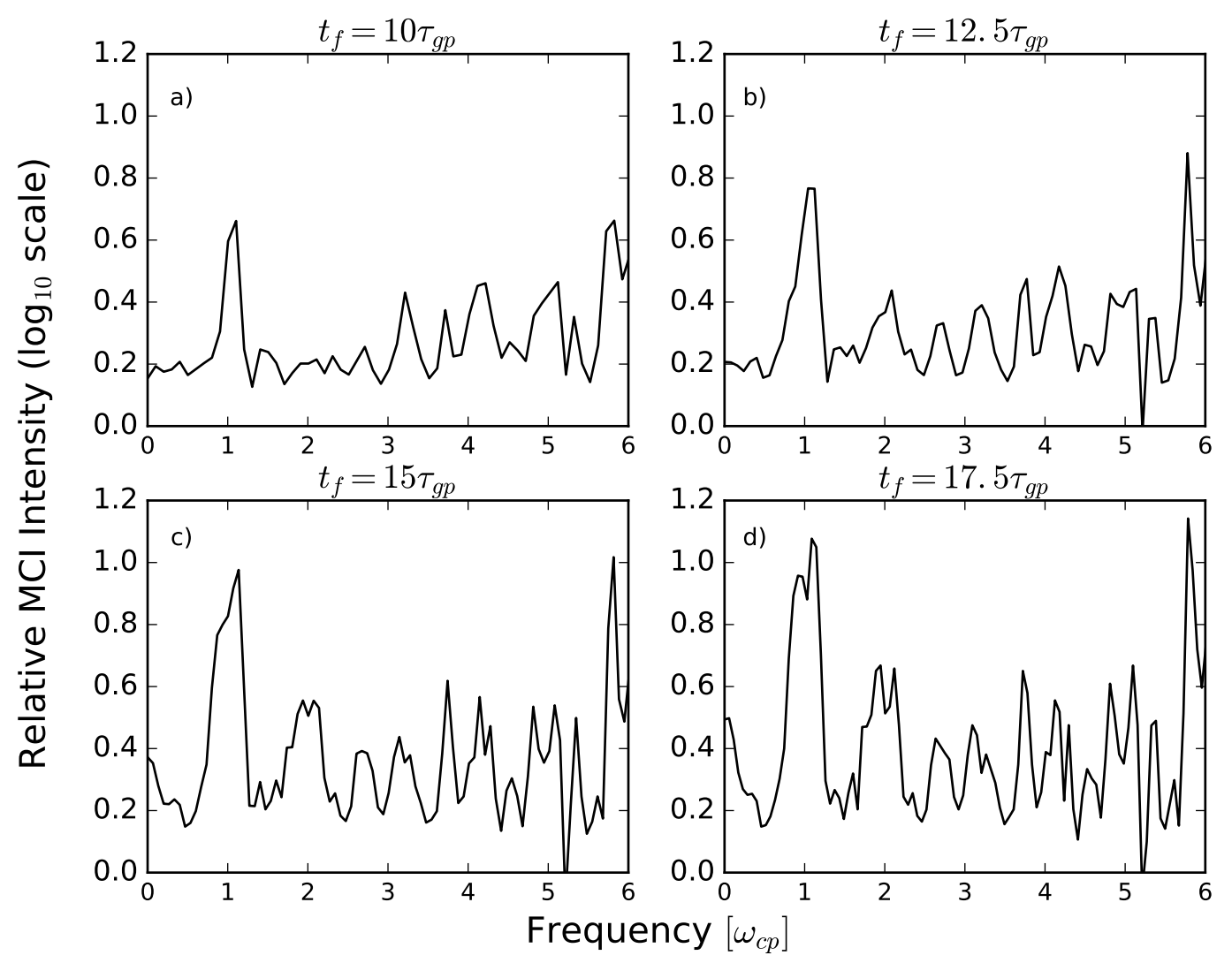

Figure 6: The difference in the $\log _{10}$ of the spectral intensity of the $B_{z}$ field component between the MCI simulation and the thermal plasma simulation, using temporal Fourier transform windows of increasing duration. Values on the y-axis denote the number of orders of magnitude by which the MCI signal exceeds the background signal. The range of data shown on the $\mathrm{x}$-axis is limited to the range of frequencies $\omega \leq 6 \omega_{c p}$, for which the spectral peaks are excited by nonlinear wave interactions. Panels a), b), c), and d) correspond to temporal Fourier transform windows of duration $10 \tau_{g p}, 12.5 \tau_{g p}, 15 \tau_{g p}$, and $17.5 \tau_{g p}$ respectively.

0 (dark blue) completely uncoupled. We use the full $17.5 \tau_{c p}$ duration of simulation data; the time integration window of each successive Fourier transform is $1.25 \tau_{c p}$, and the overlap of successive Fourier transforms is $0.25 \tau_{c p}$. The number of independent samples is thus fourteen, so that the minimum statistically significant value of $b_{c}^{2}$ is $\sim 1 / \sqrt{N} \sim 0.267$; see Appendix D of Ref. [43] for definitions and a discussion. This value of $b_{c}^{2}$ is far lower than the coupling strength of the modes of interest.

We see evidence of strong nonlinear couplings between many different modes in the simulation. Wavenumbers in the range $9 \omega_{c p} / V_{A} \leq k_{1,2} \leq 14 \omega_{c p} / V_{A}$, corresponding to the linearly unstable high amplitude modes shown in Figs. 5 and 3, are strongly coupled with each other. For example the two waves with normalised $(k, \omega) \approx(9.5,9)$ and $(k, \omega) \approx(11,10)$ can in principle interact to generate a wave with normalised $(k, \omega) \approx(1.5,1)$. This offers a possible explanation for the experimental observation of an ICE spectral peak at the fundamental proton cyclotron harmonic in the core of 

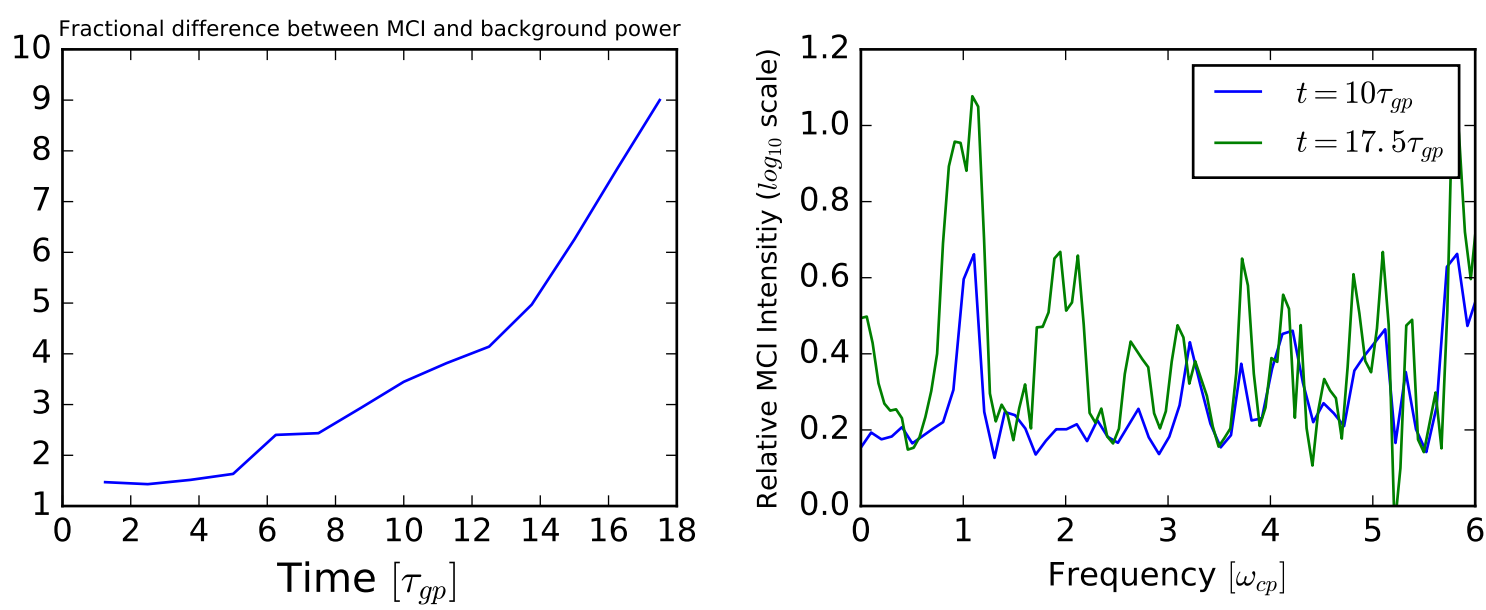

Figure 7: Left: The fractional increase in Fourier power of the $B_{z}$ field component at the $\omega=\omega_{c p}$ spectral peak of an MCI simulation and the spectral peak of a corresponding thermal plasma simulation, as a function of time. Right: the blue and green traces are identical to those of panels a) and d) of Fig. 6 respectively.

ASDEX-Upgrade, see Fig. 5 of Ref. [8]. There are numerous other wave combinations in our simulation that could also give rise to this spectral peak. The segment of Fig. 8 in the range $0.5 \lesssim k_{1} \lesssim 2,1 \lesssim k_{2} \lesssim 13$ also shows that the nonlinearly excited wave at the fundamental proton cyclotron harmonic with normalised $(k, \omega) \approx(1.5,1)$ is itself nonlinearly coupled to a range of other modes.

\section{Conclusions}

A core ICE spectral peak at the fundamental proton cyclotron frequency, equivalant to the second deuteron cyclotron harmonic frequency, has been observed in ASDEXUpgrade deuterium plasmas $[8,9,10]$. The driving population for this emission could be either fusion-born protons or NBI injected deuterons. An interpretation utilising the linear analytical theory of NBI deuterons relaxing under the MCI was given in Ref. [10]; in the present work, we have provided an alternative interpretation involving fusion-born protons and including wave excitation by nonlinear interactions. We have analysed the results of a fully kinetic PIC simulation in which the minority energetic driving population was chosen to be representative of the subset of the confined fusion-born protons found in ASDEX-Upgrade that are most likely to drive the MCI strongly. We found that the fundamental proton cyclotron harmonic was linearly stable in the simulation, but there were numerous linearly unstable MCI excited modes with $\omega>6 \omega_{c p}$. Using bicoherence analysis it was shown that these modes exhibit strong nonlinear wave-wave coupling, which gives rise to spectrally intense secondary waves in the region $\omega \leq 6 \omega_{c p}$. One such wave at $\omega=\omega_{c p}$ was observed to grow in amplitude as the simulation progressed deeper into the nonlinear regime of the MCI, and became more intense than the plasma background noise by a factor of about ten. We infer that it is entirely possible 


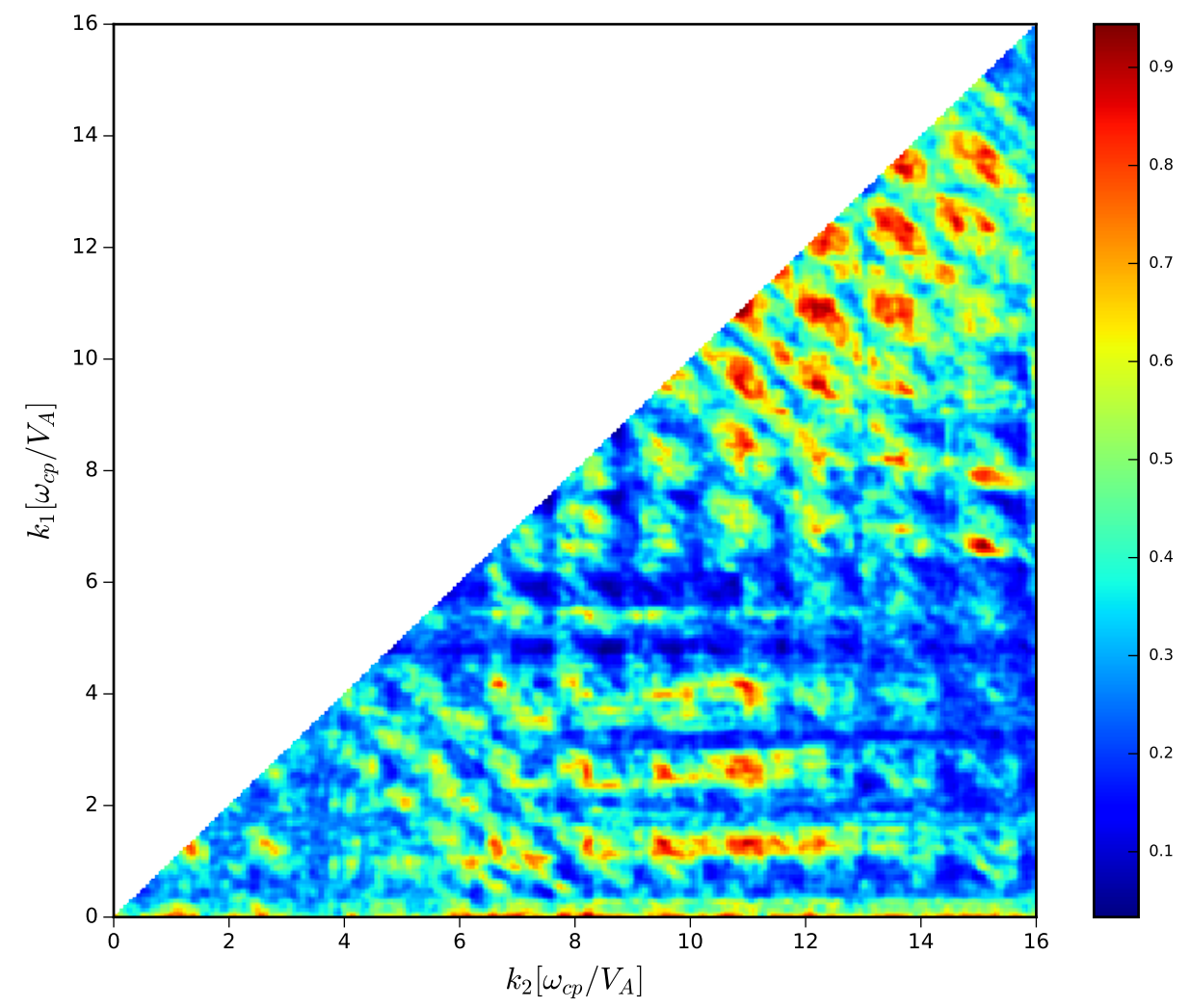

Figure 8: The square of the bicoherence $b_{c}^{2}$ of the oscillatory part of the $B_{z}$ field component as a function of normalised wavenumber $k$. The colour scale indicates the strength of intrinsic nonlinear coupling between waves with normalised wave numbers $k_{1}$ and $k_{2}$, which takes values between 0 and 1 . The value of $b_{c}^{2}$ is calculated over the full $17.5 \tau_{c p}$ duration of the simulation: the width of each successive Fourier transform is $1.25 \tau_{c p}$; and the overlap of each successive Fourier transform is $0.25 \tau_{c p}$. The number of independent samples is thus fourteen, giving a significance level of $b^{2} \gtrsim 0.267$.

that the emission at the proton fundamental, or equivalently, second deuteron cyclotron harmonic, is driven by a combination of fusion-born protons and NBI deuterons.

Future ITER burning plasmas will invariably have multiple suprathermal ion species present which may all contribute to driving ICE. ICE has been proposed as a potential diagnostic for alpha-particles in ITER, so it will be important to be able to unambiguously identify which energetic ions are responsible for the emission. It is possible that fully kinetic PIC simulations involving multiple energetic ion species may shed light on this issue in future.

In the simulations presented here, linearly excited cyclotron harmonic waves near, but below, the lower hybrid frequency were responsible for the nonlinear excitation of waves with lower frequencies, a feature previously observed in the context of JET 
plasmas in Ref. [36]. Previous MCI simulations of KSTAR plasma conditions have similarly shown that MCI excited waves in the same frequency range can also give rise to experimentally observed features above the lower hybrid frequency [41]. This excitation of waves at high and low frequencies due to nonlinear wave-wave coupling is now routinely observed in PIC simulations of the MCI such as those presented in here. This manuscript, along with the previous work outlined above, makes it clear that the nonlinear aspects of the MCI that emerge from the simulation are central to the interpretation of the observed ICE phenomena.

In both the experimental and simulation analysis of KSTAR plasma discussed in Ref. [41], the nonlinearly excited waves were less spectrally intense than the linearly excited waves to which they owe their existence. In the present manuscript, the simulation results similarly show that the nonlinearly driven wave at $\omega=\omega_{c p}$ is less spectrally intense than the linearly unstable waves which excite it. If the experimental setup of the ASDEX-Upgrade plasma we consider here was such that we able to detect these higher frequency waves, we would be able to compare the relative spectral intensity of waves with $\omega=\omega_{c p}$ and $\omega>\omega_{c p}$ between simulation and experiment. If the driving population of ICE at $\omega=\omega_{c p}$ is fusion-born protons via nonlinear interactions, then, on the basis of our simulations, there should be more intense ICE harmonics in the frequency range $\omega>\omega_{c p}$. If, however, the relative intensity of these waves does not agree between experiment and simulation, it may point towards a driving population that instead consists solely NBI deuterons or some mixture of the two species; in which case further simulations could shed light on this. Therefore, we argue that future devices such as ITER would benefit from an effort to detect higher cyclotron harmonics, such as are seen on KSTAR (see Refs. [19, 20]). In the near-term, we note that more ICE measurements spanning a large range of frequencies from a range of devices would allow deeper investigation and quantification of the above conjecture. Provided the temporal resolution of these measurements is sufficiently high, it would be possible to use bicoherence analysis to help quantify the nonlinear interactions which may lead to ICE excitation at comparatively lower frequencies. This would provide further information on the distribution and dynamics of the energetic ions, whether fusion-born or NBI, in the ICE-emitting location, whether in the core or edge plasma.

\section{Acknowledgments}

This project used the EPOCH code, part funded by UK EPSRC grants EP/G054950/1, EP/G056803/1, EP/G055165/1 and EP/ M022463/1. This work was carried out within the framework of the EUROfusion Consortium and has received funding from the Euratom research and training programme 2014-2018 and 2019-2020 under grant agreement No 633053. The work received support from the RCUK Energy Programme grant no. EP/T012250/1. The views and opinions expressed herein do not necessarily reflect those of the European Commission. 
Core proton ICE in ASDEX-Upgrade

\section{References}

[1] Cottrell G A and Dendy R O 1988 Phys. Rev. Lett. 60(1) 33-36 URL https://link.aps.org/ doi/10.1103/PhysRevLett.60.33

[2] Cottrell G A, Bhatnagar V P, Costa O D, Dendy R O, Jacquinot J, McClements K G, McCune D C, Nave M F F, Smeulders P and Start D F H 1993 Nuclear Fusion 331365 URL http://stacks.iop.org/0029-5515/33/i=9/a=I10

[3] Schild P, Cottrell G A and Dendy R O 1989 Nuclear Fusion 29834 URL http://stacks.iop. org $/ 0029-5515 / 29 / i=5 / a=013$

[4] Cottrell G A 2000 Phys. Rev. Lett. 84(11) 2397-2400 URL https://0-link-aps-org.pugwash. lib.warwick.ac.uk/doi/10.1103/PhysRevLett.84.2397

[5] Jacquet P, BergerBy G, Bobkov V, Blackman T, Day I E, Durodi F, Graham M, Hellsten T, Laxback M, Mayoral M, Monakhov I, Nightingale M, Sharapov S E, Vrancken M and contributors J E 2011 AIP Conference Proceedings 1406 17-20 URL https://aip.scitation.org/doi/ abs/10.1063/1.3664919

[6] McClements K G, Brisset A, Chapman B, Chapman S C, Dendy R O, Jacquet P, Kiptily V G, Mantsinen M, Reman B C G and Contributors J 2018 Nuclear Fusion 58096020 URL http://stacks.iop.org/0029-5515/58/i=9/a=096020

[7] Cauffman S, Majeski R, McClements K G and Dendy R O 1995 Nuclear Fusion 351597 URL http://stacks.iop.org/0029-5515/35/i=12/a=I22

[8] Ochoukov R, Bobkov V, Chapman B, Dendy R O, Dunne M, Faugel H, Garca-Muoz M, Geiger B, Hennequin P, McClements K G, Moseev D, Nielsen S, Rasmussen J, Schneider P, Weiland M and Noterdaeme J M 2018 Review of Scientific Instruments 89 10J101 URL https://doi.org/10.1063/1.5035180

[9] Ochoukov R, Bilato R, Bobkov V, Chapman B, Chapman S C, Dendy R O, Dunne M, Faugel H, Garca-Muoz M, Geiger B, Kallenbach A, Kappatou A, McClements K G, Moseev D, Nielsen S, Rasmussen J, Schneider P, Weiland M, Noterdaeme J M, Team A U and Team E M 2019 Nuclear Fusion 59014001 URL http://stacks.iop.org/0029-5515/59/i=1/a=014001

[10] Ochoukov R, McClements K G, Bilato R, Bobkov V, Chapman B, Chapman S C, Dendy R O, Dreval M, Faugel H, Noterdaeme J M, Salewski M, Weiland M, Team A U and Team E M 2019 Nuclear Fusion 59086032 URL https://doi.org/10.1088\%2F1741-4326\%2Fab2938

[11] D'Inca R 2014 Ion Cyclotron Emission on ASDEX Upgrade Ph.D. thesis Ludwig-MaximiliansUniversitat URL https://edoc.ub.uni-muenchen.de/17747/

[12] Thome K E, Pace D C, Pinsker R I, Meneghini O, del Castillo C A and Zhu Y 2018 Review of Scientific Instruments 89 10I102 URL https://doi.org/10.1063/1.5035561

[13] Thome K, Pace D, Pinsker R, Zeeland M V, Heidbrink W and Austin M 2019 Nuclear Fusion 59 086011 URL https://doi.org/10.1088\%2F1741-4326\%2Fab20e7

[14] Heidbrink W W, Austin M E, Fisher R K, García-Munoz M, Matsunaga G, McKee G R, Moyer R A, Muscatello C M, Okabayashi M, Pace D C, Shinohara K, Solomon W M, Strait E J, Zeeland M A V and Zhu Y B 2011 Plasma Physics and Controlled Fusion 53085028 URL http://stacks.iop.org/0741-3335/53/i=8/a=085028

[15] Kimura H, Kusama Y, Saigusa M, Kramer G, Tobita K, Nemoto M, Kondoh T, Nishitani T, Costa O D, Ozeki T, Oikawa T, Moriyama S, Morioka A, Fu G, Cheng C and Afanas'ev V 1998 Nuclear Fusion 381303 URL http://stacks.iop.org/0029-5515/38/i=9/a=304

[16] Ichimura M, Higaki H, Kakimoto S, Yamaguchi Y, Nemoto K, Katano M, Ishikawa M, Moriyama S and Suzuki T 2008 Nuclear Fusion 48035012 URL http://stacks. iop. org/0029-5515/48/ $i=3 / a=035012$

[17] Sato S, Ichimura M, Yamaguchi Y, Katano M, Imai Y, Murakami T, Miyake Y, Yokoyama T, Moriyama S, Kobayashi T, Kojima A, Shinohara K, Sakamoto Y, Watanabe T, Hojo H and Imai T 2010 Plasma and Fusion Research 5 S2067 URL https://doi.org/10.1585/pfr.5.S2067

[18] Liu L N, Zhang X J, Zhu Y B, Qin C M, Zhao Y P, Yuan S, Mao Y Z, Li M H, Chen Y, 
Core proton ICE in ASDEX-Upgrade

Cheng J, Ping L L, Li H and Ai L 2019 Review of Scientific Instruments 90063504 URL https://doi.org/10.1063/1.5089537

[19] Kim M, Thatipamula S, Lee J, Choi M, Park H, Akiyama T and Yun G 2018 Nuclear Fusion 58 096034 URL http://stacks.iop.org/0029-5515/58/i=9/a=096034

[20] Thatipamula S G, Yun G S, Leem J, Park H K, Kim K W, Akiyama T and Lee S G 2016 Plasma Physics and Controlled Fusion 58065003 URL http://stacks.iop.org/0741-3335/58/i=6/ $\mathrm{a}=065003$

[21] Saito K, Kasahara H, Seki T, Kumazawa R, Mutoh T, Watanabe T, Shimpo F, Nomura G, Osakabe M, Ichimura M, Higaki H and Komori A 2009 Fusion Engineering and Design 84 1676 - 1679 ISSN 0920-3796 proceeding of the 25th Symposium on Fusion Technology URL http://www.sciencedirect.com/science/article/pii/S0920379608005073

[22] Saito K, Kumazawa R, Seki T, Kasahara H, Nomura G, Shimpo F, Igami H, Isobe M, Ogawa K, Toi K, Osakabe M, Nishiura M, Watanabe T, Yamamoto S, Ichimura M, Mutoh T and Group L E 2013 Plasma Science and Technology 15209 URL http://stacks.iop.org/1009-0630/ $15 / i=3 / a=03$

[23] Shalashov A, V Suvorov E, V Lubyako L, Maassberg H and Team t 2003 Plasma Physics and Controlled Fusion 45395 URL https://doi.org/10.1088/0741-3335/45/4/306

[24] Belikov V S and Kolesnichenko Y I 1976 Sov. Phys. Tech. Phys. 201146

[25] Dendy R O, LashmoreDavies C N and Kam K F 1992 Physics of Fluids B: Plasma Physics 4 3996-4006 (Preprint https://doi.org/10.1063/1.860304) URL https://doi.org/10.1063/ 1.860304

[26] Dendy R O, LashmoreDavies C N and Kam K F 1993 Physics of Fluids B: Plasma Physics 5 1937-1944 (Preprint https://doi.org/10.1063/1.860781) URL https://doi.org/10.1063/ 1.860781

[27] Dendy R O, LashmoreDavies C N, McClements K G and Cottrell G A 1994 Physics of Plasmas 1 1918-1928 (Preprint https://doi.org/10.1063/1.870647) URL https://doi.org/10.1063/ 1.870647

[28] Dendy R O, McClements K G, LashmoreDavies C N, Majeski R and Cauffman S 1994 Physics of Plasmas 1 3407-3413 URL https://doi.org/10.1063/1.870489

[29] Dendy R O, McClements K G, Lashmore-Davies C N, Cottrell G A, Majeski R and Cauffman S 1995 Nuclear Fusion 351733 URL http://stacks.iop.org/0029-5515/35/i=12/a=I38

[30] McClements K G, Dendy R O, LashmoreDavies C N, Cottrell G A, Cauffman S and Majeski R 1996 Physics of Plasmas 3 543-553 (Preprint https://doi.org/10.1063/1.871881) URL https://doi.org/10.1063/1.871881

[31] McClements K G, Hunt C, Dendy R O and Cottrell G A 1999 Phys. Rev. Lett. 82(10) 2099-2102 URL https://link.aps.org/doi/10.1103/PhysRevLett.82.2099

[32] Fülöp T, Kolesnichenko Y, Lisak M and Anderson D 1997 Nuclear Fusion 371281 URL http://stacks.iop.org/0029-5515/37/i=9/a=I08

[33] Fülöp T and Lisak M 1998 Nuclear Fusion 38761 URL http://stacks.iop.org/0029-5515/ $38 / i=5 / a=309$

[34] Fülöp T, Lisak M, Kolesnichenko Y I and Anderson D 2000 Physics of Plasmas 7 1479-1486 (Preprint https://doi.org/10.1063/1.873967) URL https://doi.org/10.1063/1.873967

[35] Lashmore-Davies C N, Dendy R O and Kam K F 1993 Plasma Physics and Controlled Fusion 35 1529-1540 URL https://doi.org/10.1088\%2F0741-3335\%2F35\%2F11\%2F003

[36] Carbajal L, Dendy R O, Chapman S C and Cook J W S 2014 Physics of Plasmas 21012106 URL https://doi.org/10.1063/1.4861866

[37] Carbajal L, Dendy R O, Chapman S C and Cook J W S 2017 Phys. Rev. Lett. 118(10) 105001 URL https://link.aps.org/doi/10.1103/PhysRevLett.118.105001

[38] Cook J W S, Dendy R O and Chapman S C 2013 Plasma Physics and Controlled Fusion 55065003 URL http://stacks.iop.org/0741-3335/55/i=6/a=065003

[39] Cook J W S, Dendy R O and Chapman S C 2017 Phys. Rev. Lett. 118(18) 185001 URL 
https://link.aps.org/doi/10.1103/PhysRevLett.118.185001

[40] Chapman B, Dendy R O, McClements K G, Chapman S C, Yun G S, Thatipamula S G and Kim M H 2017 Nuclear Fusion 57124004 URL http://stacks.iop.org/0029-5515/57/i=12/a= 124004

[41] Chapman B, Dendy R O, Chapman S C, McClements K G, Yun G S, Thatipamula S G and Kim M H 2018 Nuclear Fusion 58096027 URL http://stacks.iop.org/0029-5515/58/i=9/ $\mathrm{a}=096027$

[42] Chapman B, Dendy R O, Chapman S C, McClements K G, Yun G S, Thatipamula S G and Kim M H 2019 Nuclear Fusion 59106021 URL https://doi.org/10.1088\%2F1741-4326\%2Fab35a7

[43] Chapman B, Dendy R O, Chapman S C, Holland L A, Irvine S W A and Reman B C G 2020 Plasma Physics and Controlled Fusion 62055003 URL https://doi.org/10.1088\%2F1361-6587\% 2Fab7a3b

[44] Reman B C G, Dendy R O, Akiyama T, Chapman S C, Cook J W S, Igami H, Inagaki S, Saito K and Yun G S 2019 Nuclear Fusion 59096013 URL https://doi.org/10.1088\%2F1741-4326\% $2 \mathrm{Fab} 2 \mathrm{ca} 2$

[45] Reman B C G, Dendy R O, Akiyama T, Chapman S C, Cook J W S, Igami H, Inagaki S, Saito K and Yun G S 2020 In preperation

[46] Birdsall C K and Langdon A B 1985 Plasma Physics Via Computer (New York, NY, USA: McGraw-Hill, Inc.) ISBN 0070053715

[47] Arber T D, Bennett K, Brady C S, Lawrence-Douglas A, Ramsay M G, Sircombe N J, Gillies P, Evans R G, Schmitz H, Bell A R and Ridgers C P 2015 Plasma Physics and Controlled Fusion 57 1-26 URL https://doi.org/10.1088/0741-3335/57/11/113001

[48] Kolesnichenko Y, Fülöp T, Lisak M and Anderson D 1998 Nuclear Fusion 381871 URL http://stacks.iop.org/0029-5515/38/i=12/a=311

[49] Kolesnichenko Y, Lisak M and Anderson D 2000 Nuclear Fusion 401419 URL http://stacks. iop.org/0029-5515/40/i=7/a=311

[50] Smith H, Fülöp T, Lisak M and Anderson D 2003 Physics of Plasmas 10 1437-1442 URL https://doi.org/10.1063/1.1566441

[51] Hellsten T and Laxback M 2003 Physics of Plasmas 10 4371-4377 URL https://doi.org/10. $1063 / 1.1617315$

[52] Hellsten T, Holmström K, Johnson T, Bergkvist T and Laxback M 2006 Nuclear Fusion 46 S442 URL http://stacks.iop.org/0029-5515/46/i=7/a=S07

[53] Smith H M and Verwichte E 2009 Plasma Physics and Controlled Fusion 51075001 URL http://stacks.iop.org/0741-3335/51/i=7/a=075001

[54] Gorelenkov N N and Cheng C Z 1995 Physics of Plasmas 2 1961-1971 (Preprint https: //doi.org/10.1063/1.871281) URL https://doi.org/10.1063/1.871281

[55] Gorelenkov N N 2016 Plasma Physics Reports 42 430-439 ISSN 1562-6938 URL https://doi. org/10.1134/S1063780X16050044

[56] Reman B C G, Dendy R O, Akiyama T, Chapman S C, Cook J W S, Igami H, Inagaki S, Saito K and Yun G S 2016 EPS Conference Proceedings P2.041 URL http://epsppd.epfl.ch/ Madeira/html/authors/nav/AutT02fr.html

[57] de Witt T D 2003 Numerical Schemes for the Analysis of Turbulence - A Tutorial (Space Plasma Simulation, Lecture Notes in Physics, vol 615) ed J. Büchner, M. Scholer, and C. T. Dum (Berlin: Springer)

[58] Kim Y C, Beall J M, Powers E J and Miksad R W 1980 The Physics of Fluids 23 258-263 URL https://aip.scitation.org/doi/abs/10.1063/1.862966

[59] Kim Y C and Powers E J 1979 IEEE Transactions on Plasma Science 7 120-131 ISSN 0093-3813

[60] Kaup D J, Reiman A and Bers A 1979 Rev. Mod. Phys. 51(2) 275-309 URL https:// 0-link-aps-org.pugwash.lib.warwick.ac.uk/doi/10.1103/RevModPhys.51.275

[61] Itoh S I, Itoh K, Nagashima Y and Kosuga Y 2017 Plasma and Fusion Research 121101003 URL https://doi.org/10.1585/pfr.12.1101003 
[62] Holland C, Tynan G R, Diamond P H, Moyer R A and Burin M J 2002 Plasma Physics and Controlled Fusion 44 A453 URL http://stacks.iop.org/0741-3335/44/i=5A/a=350

[63] Moyer R A, Tynan G R, Holland C and Burin M J 2001 Phys. Rev. Lett. 87(13) 135001 URL https://link.aps.org/doi/10.1103/PhysRevLett.87.135001

[64] Yamada T, Itoh S I, Maruta T, Kasuya N, Nagashima Y, Shinohara S, Terasaka K, Yagi M, Inagaki S, Kawai Y, Fujisawa A and Itoh K 2008 Nature Physics 4 721-725 URL https: //doi.org/10.1038/nphys1029

[65] Lee J, Yun G S, Choi M J, Kwon J M, Jeon Y M, Lee W, Luhmann N C and Park H K 2016 Phys. Rev. Lett. 117(7) 075001 URL https://link.aps.org/doi/10.1103/PhysRevLett.117. 075001 